\title{
Handling missing data in RCTs; a review of the top medical journals
}

\author{
Melanie L Bell ${ }^{1 *}$, Mallorie Fiero ${ }^{1}$, Nicholas J Horton ${ }^{2}$ and Chiu-Hsieh Hsu ${ }^{1}$
}

\begin{abstract}
Background: Missing outcome data is a threat to the validity of treatment effect estimates in randomized controlled trials. We aimed to evaluate the extent, handling, and sensitivity analysis of missing data and intention-to-treat (ITT) analysis of randomized controlled trials (RCTs) in top tier medical journals, and compare our findings with previous reviews related to missing data and ITT in RCTs.
\end{abstract}

Methods: Review of RCTs published between July and December 2013 in the BMJ, JAMA, Lancet, and New England Journal of Medicine, excluding cluster randomized trials and trials whose primary outcome was survival.

Results: Of the 77 identified eligible articles, 73 (95\%) reported some missing outcome data. The median percentage of participants with a missing outcome was $9 \%$ (range 0 - 70\%). The most commonly used method to handle missing data in the primary analysis was complete case analysis (33,45\%), while 20 (27\%) performed simple imputation, 15 (19\%) used model based methods, and 6 (8\%) used multiple imputation. 27 (35\%) trials with missing data reported a sensitivity analysis. However, most did not alter the assumptions of missing data from the primary analysis. Reports of ITT or modified ITT were found in 52 (85\%) trials, with 21 (40\%) of them including all randomized participants. A comparison to a review of trials reported in 2001 showed that missing data rates and approaches are similar, but the use of the term ITT has increased, as has the report of sensitivity analysis.

Conclusions: Missing outcome data continues to be a common problem in RCTs. Definitions of the ITT approach remain inconsistent across trials. A large gap is apparent between statistical methods research related to missing data and use of these methods in application settings, including RCTs in top medical journals.

Keywords: Missing data, Intention-to-treat, Sensitivity analysis

\section{Background}

While randomized controlled trials are considered to be the gold standard of intervention research in the biomedical setting, their validity can be threatened by missing outcome data. Participants with missing data are often a non-random subset of the sample, increasing the risk of biased estimates of treatment effects. The intention to treat (ITT) principle, in short, "analyze as randomized", is recognized as an important protection against bias by preserving the benefits of randomisation-namely balancing both known and unknown factors and eliminating selection bias $[1,2]$. When outcome data are missing, however, a true ITT analysis can be difficult or impossible to achieve, and researchers must make assumptions, some of

\footnotetext{
* Correspondence: melaniebell@email.arizona.edu

'Division of Epidemiology and Biostatistics, Mel and Enid Zuckerman College of Public Health, University of Arizona, Tucson, AZ 85724, USA

Full list of author information is available at the end of the article
}

which may be strong and unverifiable [3,4]. In the presence of incomplete data therefore, it is imperative to perform sensitivity analyses, which examine the robustness of the results to assumptions made in the primary analysis [5-8].

Missing data can reduce the power and efficiency of a study but, unfortunately, can also lead to biased results [5-7]. For example, if patients experiencing high toxicity are more likely to drop out of the trial, quality of life is likely to be overestimated and toxicity underestimated. Missing data and statistical approaches for handling them have been an active area of research and the following definitions are commonly used [9]. If missingness of the outcome of interest is unrelated to observed or unobserved patient data, the missing data are termed missing completely at random (MCAR): a strong assumption. If data are MCAR, analyzing only those with 
observed outcome data (complete case analysis) will result in some loss of efficiency but unbiased estimation [5-7]. If after taking observed data into account there are no systematic differences between participants with complete data as compared to those with missing data , data are considered to be missing at random (MAR). Multiple imputation [10] and model-based approaches, such as mixed models [11] and weighted generalized estimating equations (GEE) [12] for repeatedly measured outcomes, based on all observed data can be valid and unbiased methods for MAR data, as long as the models are specified correctly . Missing outcomes are termed missing not at random (MNAR) if systematic differences between dropouts and completers persist even after taking observed data into account. The once popular simple imputation approach of replacing missing data with the last observation carried forward (LOCF) (for longitudinal outcome data) is not necessarily valid under any of these missingness assumptions $[13,14]$. Sensitivity analyses should be performed under different assumptions than the primary analysis for example, if the primary analysis makes a MCAR assumption, the sensitivity analyses should assume MAR or MNAR.

The statistical literature is rich with methods for handling incomplete data, including approaches for sensitivity analysis and MNAR data. Guidelines for handling missing data have been published in journals such as the Journal of Clinical Oncology [15], the BMJ $[4,16,17]$ and the New England Journal of Medicine [18]. The widely adopted CONSORT statement includes a set of checklists on ITT and missing data [19]. Researchers in the field of missing data, including the Panel on Handling Missing Data in Clinical Trials commissioned recently by the National Research Council, have made calls for the use of improved methods for handling missing data including sensitivity analyses, and for more rigorous approaches to ITT analysis when outcome data are missing $[4-7,16,20]$.

Reviews of missing data and ITT in RCTs published in top medical journals for the years 1997, 2001, 2002 and 2005-2006 have been carried out [1,2,21,22]. These reviews concluded that missing outcome data in RCTs are widespread; poor handling of missing data is the norm; the term ITT is common but inconsistently used; and sensitivity analyses are rarely (if ever) reported. With the recent guidelines and exhortations to more appropriately handle missing data (including planning and prevention), we hypothesized that the amount of missing data would have decreased and current approaches would have improved. The aims of this study, therefore, were to identify, in RCTs published in the top medical journals, the proportion of trials:

1. with missing data and their handling of missing data;

2. reporting sensitivity analyses regarding missing data; and

3. reporting an intention to treat analysis.
Secondary aims included assessing indicators of planning for and prevention of, missing outcome data, and to compare current approaches with those reported in the previous reviews.

\section{Methods}

We performed a PubMed search of randomized controlled trials published in four top medical journals: the British Medical Journal (BMJ); Journal of the American Medical Association (JAMA); The Lancet; and New England Journal of Medicine (NEJM) between July and December 2013. Cluster randomized trials and trials whose primary outcomes were survival were excluded because 1) the statistical issues for these are different to those in individually randomized and for non-survival outcomes and 2) we wanted to compare our results to a previous review (see below). The search strategy included searching for studies in each journal whose publication type was classified as "randomized controlled trials". We examined each paper collected from the initial search and identified relevant studies based on study exclusion criteria.

\section{Content assessment}

All articles were assessed by one reviewer (MF) using a standardized form, and $15 \%$ of the studies were randomly selected to be independently assessed by a second and third reviewer. We calculated kappa statistics to evaluate inter-rater reliability for methods used to handle missing data in primary analysis, whether an intention-to-treat analysis was performed, and sensitivity analysis. All disagreements were resolved by consensus.

\section{Extent and handling of missing data}

For each article we determined the magnitude of missing data and method(s) for handling missing data in the principal analysis. We defined the number of subjects per trial as the number of subjects randomized. The proportion with a missing outcome was computed as the number of subjects with a missing outcome divided by the total number of subjects randomized. The principal analysis was defined as the main analysis performed on the primary outcome. When more than one primary outcome was reported in the trial, we used the outcome that appeared first in the methods section. For primary outcome measurements monitored repeatedly, we used the final follow-up time point to calculate the missing rate, unless a preceding time point was specified. We identified the statistical method used to handle missing data in the principal analysis and classified these as complete case, simple imputation (such as last or worst observation carried forward), multiple imputation or model based (for example, mixed models or generalized estimating equations). Complete case was defined as using only individuals who had complete primary outcome data for the stated primary analysis. To assess 
prevention and planning, we recorded whether mention was made of attempts to avoid missing data, whether sample size calculations accounted for missing data, and by how much observed and expected attrition rates differed.

\section{Sensitivity analysis for missing data}

We assessed method(s) to deal with missing data in any sensitivity analysis and calculated the proportion of trials that reported carrying out a sensitivity analysis. We defined sensitivity analysis as any alternative technique performed to further investigate the effects of missing outcome data on primary results.

\section{Intention-to-treat analysis}

We determined the proportion of trials that reported an ITT or modified ITT analysis, and verified whether all randomized subjects were analyzed and how missing data were handled if any.

\section{Comparison of reviews on missing data}

We compared our findings with previous reviews of missing data and ITT in RCTs. For each review we reported, if possible, the number of trials: included in the review, with missing data, reporting sensitivity analysis, reporting ITT, and missing data approaches. We aimed to compare our results directly to Wood et al, [16] to assess whether changes have occurred since their 2004 paper on RCTs published in 2001. We therefore used similar definitions, inclusion criteria and collected data in a similar fashion. We used chi-square tests for comparisons. Other reviews were compared qualitatively.

\section{Results}

Our search identified 148 randomized controlled trials published within the six-month period. A total of 71 trials were excluded (19 were cluster randomized controlled trials, 52 had a primary outcome as time to event), leaving 77 articles to review. Inter-rater agreement for methods used to handle missing data in primary analysis, intentionto-treat, and sensitivity analysis were $0.72,0.94$, and 0.78 , respectively, showing "substantial" to "near perfect" agreement, according to Landis and Koch [23]. Table 1 shows the general characteristics of the included trials. Together, the median number of subjects randomized was 368 , with a range of $13-53,450$. A list of included studies can be found in Additional file 1.

\section{Extent and handling of missing data}

Seventy-three (95\%) trials reported some missing outcome data. The median percentage of patients with a missing outcome was $9 \%$, with a range of $0-70 \%$. Sixty-six trials reported reasons why outcomes were missing with reasons ranging from simply stating that patients were lost to follow-up to very specific explanations. The majority of
Table 1 General characteristics of the 77 randomized controlled trials published July - December 2013

\begin{tabular}{lc}
\hline & N (\%) \\
\hline Journal & $8(10)$ \\
BMJ & $26(34)$ \\
Lancet & $22(29)$ \\
NEJM & $21(27)$ \\
Number of centers involved & \\
$\quad$ Single & $21(27)$ \\
$\quad$ Multiple & $56(73)$ \\
Type of outcome & \\
$\quad$ Quantitative & $42(55)$ \\
$\quad$ Binary & $35(45)$ \\
How often outcome was collected & \\
$\quad$ Single & $16(21)$ \\
Repeated & $61(79)$ \\
How outcome was treated in the primary analysis & \\
$\quad$ Single & $63(82)$ \\
Repeated & $14(18)$ \\
Reported CONSORT flow diagram & $76(99)$ \\
Reported primary analysis was intention-to-treat or \\
modified intention-to-treat
\end{tabular}

trials reported these details in their CONSORT flow diagram [19].

Complete case analysis was the most common method to handle missing data $(33,45 \%)$. Twenty $(27 \%)$ trials used simple imputation methods. Three (5\%) used linear interpolation, eight (11\%) used worst-case imputation, nine $(12 \%)$ used last observation carried forward. Six (8\%) trials performed multiple imputation. Fifteen (19\%) trials used model based methods: 11 (15\%) used mixed models and three (4\%) used un-weighted generalized estimating equations (Table 2). Thus, a MAR assumption for the primary analysis was made in 17 (23\%) of the trials with missing data.

Of the trials reporting more than $10 \%$ missing data, 13 (36\%) used complete case, 13 (36\%) used simple imputation, 3 (8\%) used multiple imputation, and 7 (19\%) used model based methods. Of the trials reporting less than $10 \%$ missing data, 20 (54\%) used complete case, 7 (19\%) used simple imputation, 3 (8\%) used multiple imputation, and 7 (19\%) used model based methods.

Sixty-six (86\%) trials presented a sample size calculation, with 38 of them accounting for missing data in the calculation by inflating the sample size by one minus the expected attrition rate. The mean absolute difference in the actual attrition rate and the expected was $8 \%$ with a range of $0.3-$ $31 \%$. Two trials accounting for missing data in the sample 
Table 2 Handling of missing data in primary analysis among 73 trials who reported missing outcome data

\begin{tabular}{lc}
\hline & $\mathbf{N ~ ( \% ) ^ { 1 }}$ \\
\hline Proportion of patients with missing outcome & \\
No missing data & $4(5)$ \\
$0-1 \%$ & $2(3)$ \\
$1-5 \%$ & $11(14)$ \\
$5-10 \%$ & $24(31)$ \\
$>10 \%$ & $36(47)$ \\
Reported number of patients with missing outcomes & $71(97)$ \\
by randomized treatment arm & $66(90)$ \\
Reported reasons why missing & $26(36)$ \\
$\begin{array}{l}\text { Mentioned attempts to avoid missing data before } \\
\text { and during trial }\end{array}$ & \\
Methods & $33(45)$ \\
Complete case & $3(5)$ \\
Simple imputation & $8(11)$ \\
Linear interpolation & $9(12)$ \\
Worst-case & $6(8)$ \\
LOCF & $3(4)$ \\
Multiple imputation & $11(15)$ \\
Model based & \\
GEE (un-weighted) & \\
Mixed model/hierarchical/multilevel & \\
the other denominators are 73 (the number of studies with any missing data).
\end{tabular}

size calculation had unclear expected attrition rates. $72 \%$ estimated higher attrition rates than observed, while $28 \%$ estimated lower attrition rates than observed.

Attempts to avoid missing data before and during the trial were mentioned in 26 trials. The median percentage of missing data for those who mentioned attempts to avoid missing data was $12 \%$, with a range of $2-56 \%$. The median percentage of missing data for those who did not mention attempts to avoid missing data was $9 \%$, with a range of $0.6-70 \%$.

Seventy-one (97\%) trials reported the number of patients with missing outcome by treatment arm. Nine trials reported comparisons of baseline characteristics between patients with observed and missing outcomes. Six of them reported a significant difference.

\section{Sensitivity analysis for missing data}

Twenty-seven trials (35\%) reported performing a sensitivity analysis with respect to missing data (Table 3). Of these, ten $(37 \%)$ trials used multiple imputation in the sensitivity analysis. Six (22\%) performed a complete case analysis. Four (15\%) trials carried out simple imputation: two performed worst-case imputation and one imputed with baseline value. One trial performed both complete case analysis and LOCF. One trial performed worst-case imputation, LOCF, and multiple imputation. Two trials carried out adjustments using auxiliary data. One trial used un-weighted GEE and two trials used mixed models. Two trials reported that they performed a sensitivity analysis, but methods were unclear.

In total, 11 made a MCAR assumption for the sensitivity analysis, and 15 made a MAR assumption. Only 10 weakened the missingness assumption of the primary analysis to perform their sensitivity analysis, by using a MCAR assumption for the primary followed by a MAR assumption in the sensitivity. No researchers used MNAR models. Of the 36 trials reporting more than $10 \%$ missing data, 16 (44\%) performed a sensitivity analysis: 4 used complete case, 2 used simple imputation, 1 used simple and multiple imputation, 6 used multiple imputation, 2 made adjustments using auxiliary data, and 1 approach was unclear. Of the 41 trials reporting less than 10\% missing data, 11 (27\%) performed a sensitivity analysis: 2 used complete case, 1 used complete case and simple imputation, 1 used simple imputation, 3 used multiple imputation, 3 used model based methods, and 1 was unclear.

\section{Intention-to-treat analysis}

Fifty-two reported the use of ITT and 14 reported the use of modified ITT for their primary analysis. Definitions of ITT and modified ITT differed across trials. Of the articles that reported ITT or modified ITT analysis, 21 (40\%) included all randomized subjects in the primary analysis. Of the articles that reported ITT or modified ITT analysis, 62 (94\%) had missing data. Of the trials with missing data, 48 (66\%) reported ITT analysis and 14 (19\%) reported modified ITT analysis.

\section{Comparison of reviews on missing data}

Of the five reviews that we considered, including our own, missing data rates were fairly similar, ranging from 61$95 \%$. Use of complete case analysis and simple imputation were consistent across reviews, ranging from $45-65 \%$ and $17-27 \%$, respectively. Recently, there has been an increase in application of multiple imputation and model based methods for missing data in primary analyses. The number of papers reporting sensitivity analysis for missing data (1-37\%) and ITT analysis (48-85\%) have both increased across time. See Table 4.

In comparison to Wood et al.'s [22] review of RCTs from 2001, trials reporting missing data were similar: $89 \%$ versus $95 \%$ (95\% confidence interval (CI) for difference $-3 \%$ to $15 \% ; \mathrm{P}=0.18$ ). The percentage of papers with $10 \%$ or more missing data was also similar, at $51 \%$ versus $47 \%$ (95\% CI for difference $-12 \%$ to $20 \%$; $\mathrm{P}=$ 0.63). Of those that reported missing data, trials that used complete case analysis decreased from $65 \%$ to $45 \%$ (95\% CI for difference $-36 \%$ to $-3 \%$; $\mathrm{P}=0.02$ ). The 
Table $\mathbf{3}$ Methods for handling missing data in sensitivity analysis in $\mathbf{2 7}$ trials

\begin{tabular}{|c|c|c|c|c|c|}
\hline Sensitivity method & Assumption & Primary analysis & Assumption & $\mathbf{N}$ & Total N (\%) \\
\hline \multirow[t]{3}{*}{ Complete case } & MCAR & Simple imputation & MCAR & 3 & $6(22)$ \\
\hline & & $\mathrm{Ml}$ & MAR & 2 & \\
\hline & & Mixed Model & MAR & 1 & \\
\hline \multirow[t]{3}{*}{ Simple imputation ${ }^{1}$} & MCAR & GEE & MCAR & 1 & $4(15)$ \\
\hline & & $\mathrm{Ml}$ & MAR & 1 & \\
\hline & & Mixed model & MAR & 2 & \\
\hline GEE (un-weighted) & MCAR & Complete case & MCAR & 1 & $1(4)$ \\
\hline \multirow[t]{4}{*}{$M 1^{2}$} & MAR & Complete case & MCAR & 6 & $10(37)$ \\
\hline & & Simple imputation & MCAR & 1 & \\
\hline & & GEE & MCAR & 1 & \\
\hline & & Mixed model & MAR & 2 & \\
\hline Mixed model & MAR & Complete case & MCAR & 2 & $2(7)$ \\
\hline Adjustment using auxiliary data & MAR & Mixed model & MAR & 2 & $2(7)$ \\
\hline \multirow[t]{2}{*}{ Unclear } & & Complete case & MCAR & 1 & $2(7)$ \\
\hline & & Simple imputation & MCAR & 1 & \\
\hline
\end{tabular}

${ }^{1}$ One trial also performed complete case analysis.

${ }^{2}$ One trial also performed simple imputation.

percent that performed simple imputation was similar, at $22 \%$ as compared to $27 \%$ (95\% CI for difference $-9 \%$ to $19 \% ; \mathrm{P}=0.49$ ). The use of multiple imputation or model based methods increased from $10 \%$ to $27 \%$ (95\% CI for difference $5 \%$ to $30 \%$; $\mathrm{P}=0.01$ ). Mentions of attempts to avoid missing data was similar, at $35 \%$ compared to $29 \%$ (95\% CI for difference $-46 \%$ to $-58 \%$; $\mathrm{P}=0.83$ ). Sensitivity analysis for missing data increased from $21 \%$ to $37 \%$ (95\% CI for difference $1 \%$ to $31 \%$; $\mathrm{P}=0.04$ ). Reports of ITT or modified ITT analysis increased from $41 \%$ to $85 \%$ (95\% CI for difference $29 \%$ to $59 \%$; P < 0.0001 ).

\section{Discussion}

\section{Summary}

Our review of 77 RCTs published in the top medical journals found that $95 \%$ of trials reported some missing outcome data, with a median of $9 \%$, and up to $70 \%$. Complete case analysis was the most common way of handling missing data in the primary analysis (45\%), followed by simple imputation (27\%), model based methods (mixed models and un-weighted generalized estimating equations) (19\%) and multiple imputation (8\%). Sensitivity analyses were performed in $35 \%$ of the trials, but most (63\%) did not weaken the assumptions regarding missing data from their primary analysis. An ITT or modified ITT was reported in $85 \%$ of the trials. Most reports included a sample size calculation (86\%), and $58 \%$ of these inflated the sample size to account for expected attrition. These calculations tended to be conservative, with $72 \%$ estimating higher dropout than observed with a difference of $8 \%$, and ranging up to $30 \%$ higher.

\section{Relation to other literature}

The amount of missing data appears to have remained fairly constant over time, as does the proportion of trials that mentioned attempts to avoid missing data. While it is possible that those trials that did not report prevention attempts did, in fact, employ them, it may be that researchers need to give more consideration to missing data during trial design and conduct.

The use of methods with the strong assumption that data are missing completely at random (complete case analysis, simple imputation and un-weighted GEE) has remained popular: $85 \%$ in the current review as compared to $89 \%$ in the 2001 review. This is in direct contrast to recommendations put forth by leaders in the field, including the National Research Council's Committee on National Statistics (CNSTAT) Panel on Handling Missing Data in Clinical Trials which recommends a primary analysis that assumes data are missing at random, followed by sensitivity analyses which weaken this assumption and allows for data not missing at random $[7,18]$. White et al. [4] suggest a four part strategy: 1) attempt to follow up all subjects; 2) carry out a primary analysis of all observed data that are valid under a plausible assumption; 3) perform sensitivity analyses to explore the effect of departures from the primary assumption; and 4) account for all randomized participants in at least one of the analyses. This approach was utilized in the analysis of an alcohol screening and brief intervention study [24]. It was discouraging that sensitivity analyses which contradict the assumptions of the primary analyses remain so rare. 
Table 4 Reviews on missing data and ITT in the BMJ, JAMA, NEJM and the Lancet ${ }^{1}$

\begin{tabular}{|c|c|c|c|c|c|c|c|c|c|}
\hline Study & Timing & Study inclusion criteria & $\begin{array}{l}\text { Number of } \\
\text { trials included }\end{array}$ & $\begin{array}{l}\text { Number (\%) of } \\
\text { papers with } \\
\text { missing data }{ }^{2}\end{array}$ & $\begin{array}{l}\text { Number (\%) of papers } \\
\text { with more than } 10 \% \\
\text { missing data }^{2}\end{array}$ & $\begin{array}{l}\text { Missing data } \\
\text { approaches in } \\
\text { primary analysis }\end{array}$ & Number $(\%)^{3}$ & $\begin{array}{l}\text { Number (\%) of } \\
\text { papers reporting } \\
\text { sensitivity analysis }\end{array}$ & $\begin{array}{l}\text { Number (\%) of } \\
\text { papers reporting } \\
\mathrm{ITT}^{4} \\
\end{array}$ \\
\hline \multirow{5}{*}{$\begin{array}{l}\text { Hollis et al., } \\
1999 \text { [2] }\end{array}$} & \multirow[t]{5}{*}{1997} & \multirow[t]{5}{*}{ All RCTs } & \multirow[t]{5}{*}{249} & \multirow[t]{5}{*}{$89 / 119(75)$} & \multirow[t]{5}{*}{ 29/119 (24) } & Complete case & $44(49)$ & \multirow[t]{5}{*}{$1(1)$} & \multirow[t]{5}{*}{ 119/249 (48) } \\
\hline & & & & & & Simple imputation & $15(17)$ & & \\
\hline & & & & & & Multiple imputation & 0 & & \\
\hline & & & & & & Model based & $29(33)$ & & \\
\hline & & & & & & Unclear & $1(1)$ & & \\
\hline \multirow{5}{*}{$\begin{array}{l}\text { Wood et al., } \\
2004 \text { [22] }\end{array}$} & \multirow[t]{5}{*}{ July-Dec, 2001} & \multirow{5}{*}{$\begin{array}{l}\text { All RCTs with non-survival } \\
\text { outcomes }\end{array}$} & \multirow[t]{5}{*}{71} & \multirow[t]{5}{*}{ 63/71 (89) } & \multirow[t]{5}{*}{$36 / 71(51)$} & Complete case & $41(65)$ & \multirow[t]{5}{*}{$13(21)$} & \multirow[t]{5}{*}{$26 / 63(41)$} \\
\hline & & & & & & Simple imputation & $14(22)$ & & \\
\hline & & & & & & Multiple imputation & $1(2)$ & & \\
\hline & & & & & & Model based & $5(8)$ & & \\
\hline & & & & & & Unclear & $2(3)$ & & \\
\hline \multirow{5}{*}{$\begin{array}{l}\text { Gravel et al., } \\
2007 \text { [1] }\end{array}$} & \multirow[t]{5}{*}{2002} & \multirow[t]{5}{*}{ Sample of RCTs ${ }^{1}$} & \multirow[t]{5}{*}{403} & \multirow[t]{5}{*}{$152 / 249(61)$} & \multirow[t]{5}{*}{$52 / 249(21)$} & Complete case & $89(59)$ & \multirow[t]{5}{*}{ Not reported } & 249/403 (62) \\
\hline & & & & & & Simple imputation & $32(21)$ & & $201 / 283(71)^{5}$ \\
\hline & & & & & & Multiple imputation & $1(1)$ & & \\
\hline & & & & & & Model based ${ }^{6}$ & 0 & & \\
\hline & & & & & & Unclear $^{6}$ & $30(20)$ & & \\
\hline \multirow{5}{*}{$\begin{array}{l}\text { Fielding et al., } \\
2008 \text { [21] }\end{array}$} & \multirow[t]{5}{*}{$2005-2006$} & \multirow{5}{*}{$\begin{array}{l}\text { Random sample of RCTs } \\
\text { with Quality of life } \\
\text { outcomes }\end{array}$} & \multirow[t]{5}{*}{61} & \multirow[t]{5}{*}{$55 / 61(90)$} & \multirow[t]{5}{*}{ 22/61 (36) } & Complete case & $30(55)$ & \multirow[t]{5}{*}{$6(11)$} & \multirow[t]{5}{*}{ Not reported } \\
\hline & & & & & & Simple imputation & $11(20)$ & & \\
\hline & & & & & & Multiple imputation & $1(2)$ & & \\
\hline & & & & & & Model based & $9(16)$ & & \\
\hline & & & & & & Unclear & $4(7)$ & & \\
\hline \multirow{4}{*}{$\begin{array}{l}\text { Bell et al., } \\
\text { (current study) }\end{array}$} & July-Dec, 2013 & All RCTS with non-survival & 77 & 73/77 (95) & $36 / 77(47)$ & Complete case & $33(45)$ & $27(37)$ & $62 / 73(85)$ \\
\hline & & outcomes & & & & Simple imputation & $20(27)$ & & \\
\hline & & & & & & Multiple imputation & $6(8)$ & & \\
\hline & & & & & & Model based & $14(19)$ & & \\
\hline
\end{tabular}

'Gravel et al. reported on 10 journals, including the BMJ, JAMA, NEJM and the Lancet.

2Denominator is the number of trials included except for Hollis et al. and Gravel et al., where denominators are the number of papers reporting ITT.

${ }^{3}$ Denominator is the number of papers with missing data.

${ }^{4}$ Denominator is the number of papers with missing data except for Hollis et al. and Gravel et al. where denominators are the number of trials included in the review.

${ }^{5}$ Sub-analysis of RCTs from the four journals (BMJ, JAMA, NEJM and the Lancet) out of the 10 journals included in Gravel's review.

${ }^{6}$ Three reported as "other" might be model based (added to 27 marked "unclear"). 
The CNSTAT report favored inverse probability weighted generalized estimating equation (GEE) methods and multiple imputation, in part because auxiliary data associated with missingness can be incorporated into the analysis [18]. However, none of the RCTs in our review used weighted GEEs, and only 8 used MI for the primary analysis. While the number of trials reporting sensitivity analyses appear to have increased over time, from $1 \%$ in $1997,21 \%$ in 2001 , and up to $37 \%$ in our review, none of the studies reported using MNAR models, or appeared to follow the Panel's guidelines for sensitivity analyses. Perhaps the reluctance to use more sophisticated approaches is due to a lack of knowledge or experience on the parts of applied researchers and/or biostatisticians. Perhaps it is due to the time lag between reports of methods and software to implement them.

Reports of ITT or modified ITT analysis have increased substantially over time, from $48 \%$ in $1997,41 \%$ in 2001 , $71 \%$ in 2007 , to $85 \%$ in 2013 . However, we found, as others have $[1,2]$, that these terms are used inconsistently: only $40 \%$ actually included all randomized participants in the primary analysis. White et al. [20] call for at least one analysis (primary or sensitivity) to include all participants. This lack of consistent definition (even amongst methodologists [25]) and clarity regarding who was included in the analysis has led the CONSORT statement's authors to remove the ITT request in their 2010 update (over the original 2001 statement) [19].

Many trials had repeated measurements, (79\%) but only 14 of these used all the measurements in the primary analysis, often resulting in a strong MCAR assumption. Using all outcome data, even if the primary interest is in a specific time point, can reduce some or all of the bias due to data which are missing non-randomly. For example, Bell and Fairclough use several methods to analyze quality of life measured at four time points in an RCT with substantial missing data. A t-test comparing the two arms at the $4^{\text {th }}$ timepoint found a treatment effect of -0.4 ; a contrast from a mixed model estimated the effect to be -8.0 [5]. Simulation studies have demonstrated the bias that can occur when a MCAR assumption is made for data which are MAR $[17,26]$. Approaches which use all repeated measures data and are valid for MAR data include multiple imputation, mixed models, inverse probability weighted GEEs, and Bayesian analysis [5-7].

\section{Strengths and limitations}

A strength of our review is the inclusion of other reviews to assess possible time trends. In particular, we followed Wood and colleagues [22] methods and definitions in order to make direct comparisons between 2001 and 2013. A limitation is the difficulty in making comparisons with the other reviews, due to different inclusion criteria. For example, inclusion of survival outcomes may reduce missing data rates, as participants who drop out are often considered censored. Different definitions may also hinder comparisons. For example, the rate of missing data when measurements are taken repeatedly could be: 1 ) the number of patients with any missing primary outcome data divided by the total number of patients randomized; or 2) the number of missing assessments divided by the total number of assessments. We chose the former, in line with Wood et al. [22] Another limitation is that we focused on the top 4 medical journals. It is likely that trial reports appearing in these journals have higher standards of conduct and reporting, so that this review may underestimate the extent of missing data and overestimate the use of sensitivity analyses.

\section{Recommendations and conclusion}

We have several recommendations. First, missing data should be considered at each stage of a trial: design, conduct, analysis, and reporting. Prevention is the best way to handle missing data, so more effort needs to be put into missing data at the design and conduct stage. The CNSTAT report [7], and it's synopsis [18] discuss several approaches. Second, we recommend that in trials with repeated measurements, all data should be used in an analysis that makes a plausible assumption about missing data. Usually this will be a MAR assumption. Third, sensitivity analyses that weaken the assumptions about missing data should be carried out and reported. For example, if the primary analysis uses a MAR assumption, the sensitivity analysis should assume MNAR.

There appears to be a large gap in translation between statistical methods research and the use of these methods in applications, such as RCTs. For example, simple imputation remains popular, despite warnings from many statisticians against their use, particularly LOCF [5-7,13,14,27-29]. This failure to translate persists, despite papers regarding missing data, sensitivity analyses, and strategies for intention to treat in the presence of missing data being published in high impact medical journals [4,16-18]. More statisticians should attempt to make their work accessible to applied researchers, by publishing secondary papers in appropriate applied journals showing how to make their methods work in practice. Applied statisticians and researchers should read these papers to update their skillsets and use modern methods that increase statistical power and in some cases reduce bias. Editors and reviewers should demand that modern methods which use all the data are used, at least in the sensitivity analysis.

\section{Conclusions}

Applied researchers and statisticians need to improve their handling of missing data in RCTs. 


\section{Additional file}

Additional file 1: References of the 77 trials included in the missing data in RCTs review.

\section{Abbreviations}

ITT: Intention to treat; MCAR: Missing completely at random; MAR: Missing at random; MNAR: Missing not at random; GEE: Generalized estimating equations; LOCF: Last observation carried forward; RCT: Randomized controlled trial; MI: Multiple imputation; Cl: Confidence interval.

\section{Competing interests}

The authors declare that they have no competing interests.

\section{Authors' contributions}

MB conceived the idea, directed the research, contributed to analysis and reporting and is the guarantor. MF collected and analyzed data, and contributed to the reporting. $\mathrm{CHH}$ and $\mathrm{NJH}$ contributed to analysis and reporting. All authors read and approved the final manuscript.

\section{Authors' information}

Melanie L Bell is Associate Professor of Biostatistics. Mallorie Fiero is a PhD candidate in Biostatistics. Nicholas J Horton is Professor of Biostatistics. Chiu-Hsieh Hsu is Associate Professor of Biostatistics.

\section{Author details}

'Division of Epidemiology and Biostatistics, Mel and Enid Zuckerman College of Public Health, University of Arizona, Tucson, AZ 85724, USA. ²Department of Mathematics and Statistics, Amherst College, Amherst, MA 01002, USA.

\section{Received: 11 July 2014 Accepted: 30 October 2014}

Published: 19 November 2014

\section{References}

1. Gravel J, Opatrny L, Shapiro S: The intention-to-treat approach in randomized controlled trials: are authors saying what they do and doing what they say? Clin Trials 2007, 4(4):350-356

2. Hollis $\mathrm{S}$, Campbell F: What is meant by intention to treat analysis? survey of published randomised controlled trials. Br Med J 1999, 319(7211):670-674.

3. Altman DG: Missing outcomes in randomized trials: addressing the dilemma. Open Medicine 2009, 3(2):e51-e53.

4. White IR, Horton NJ, Carpenter J, Pocock SJ: Strategy for intention to treat analysis in randomised trials with missing outcome data. BMJ 2011, 342:d40.

5. Bell ML, Fairclough DL: Practical and statistical issues in missing data for longitudinal patient reported outcomes. Stat Methods Med Res 2014 23(5):440-459.

6. Carpenter J, Kenward M: Missing Data in Randomised Controlled Trials - a Practical Guide. Birmingham: National Institute for Health Research; 2008. vol. Publication RM03/JH17/MK

7. Council NR: The prevention and treatment of missing data in clinical trials. In Committee on National Statistics, Division of Behavioral and Social Sciences and Education. Washington DC: National Academies Press; 2010.

8. Thabane L, Mbuagbaw L, Zhang S, Samaan Z, Marcucci M, Ye C, Thabane M, Giangregorio L, Dennis B, Kosa D, Deono V, Dillenburg R, Fruci V, Bawor M, Lee J, Wells G, Goldsmith C: A tutorial on sensitivity analyses in clinical trials: the what, why, when and how. BMC Med Res Methodol 2013, 13(1):92.

9. Little R, Rubin D: Statistical Analysis With Missing Data. 2nd edition Hoboken, NJ: Wiley; 2002

10. Rubin DB: Multiple Imputation for Nonresponse in Surveys. New York: J Wiley \& Sons; 1987.

11. Fitzmaurice GM, Laird NM, Ware JH: Applied Longitudinal Analysis. 2nd edition. Hoboken NJ: Wiley; 2011.

12. Robins JM, Rotnitzky A, Zhao LP: Analysis of semiparametric regression models for repeated outcomes in the presence of missing data. J Am Stat Assoc 1995, 90:106-121.

13. Mallinckrodt CH, Watkin JG, Molenberghs G, Carroll RJ: Choice of the primary analysis in longitudinal clinical trials. Pharm Stat 2004, 3(3):161-169.
14. Molenberghs G, Thijs H, Jansen I, Beunckens C, Kenward MG, Mallinckrodt C, Carroll RJ: Analyzing incomplete longitudinal clinical trial data. Biostatistics 2004, 5(3):445-464.

15. Ibrahim JG, Chu H, Chen M-H: Missing data in clinical studies: issues and methods. J Clin Oncol 2012, 30(26):3297-3303.

16. Sterne J, White I, Carlin J, Spratt M, Royston P, Kenward M, Wood A Carpenter J: Multiple imputation for missing data in epidemiological and clinical research: potential and pitfalls. BMJ 2009, 338:b2393.

17. Bell ML, Kenward MG, Fairclough DL, Horton NJ: Differential dropout and bias in RCTs: when it matters and when it may not. Br Med J 2013, 346:e8668.

18. Little RJ, D'Agostino R, Cohen ML, Dickersin K, Emerson SS, Farrar JT, Frangakis C, Hogan JW, Molenberghs G, Murphy SA, Neaton JD, Rotnitzky A, Scharfstein D, Shih WJ, Siegel JP, Stern H: The prevention and treatment of missing data in clinical trials. New England J Med 2012, 367(14):1355-1360.

19. Moher D, Hopewell S, Schulz KF, Montori V, Gøtzsche PC, Devereaux PJ, Elbourne D, Egger M, Altman DG: CONSORT 2010 explanation and elaboration: updated guidelines for reporting parallel group randomised trials. BMJ 2010, 340:c869.

20. White IR, Carpenter J, Horton NJ: Including all individuals is not enough: lessons for intention-to-treat analysis. Clin Trials 2012, 9:396-407.

21. Fielding S, Maclennan G, Cook JA, Ramsay CR: A review of RCTs in four medical journals to assess the use of imputation to overcome missing data in quality of life outcomes. Trials 2008, 9:51.

22. Wood AM, White IR, Thompson SG: Are missing outcome data adequately handled? a review of published randomized controlled trials in major medical journals. Clin Trials 2004, 1(4):368-376.

23. Landis JR, Koch GG: The measurement of observer agreement for categorical data. Biometrics 1977, 33(1):159-174.

24. Kypri K, Vater T, Bowe SJ, Saunders JB, Cunningham JA, Horton NJ, McCambridge J: Web-based alcohol screening and brief intervention for university students: a randomized trial. JAMA 2014, 311(12):1218-1224.

25. Alshurafa M, Briel M, Akl EA, Haines T, Moayyedi P, Gentles SJ, Rios L, Tran C, Bhatnagar N, Lamontagne F: Inconsistent definitions for intention-to-treat in relation to missing outcome data: systematic review of the methods literature. PLoS One 2012, 7(11):e49163.

26. Bell ML, Fairclough DL, King MT: Bias in area under the curve for longitudinal clinical trials with missing patient reported outcome data: summary measures versus summary statistics. 2014.

27. Beunckens C, Molenberghs G, Kenward MG: Direct likelihood analysis versus simple forms of imputation for missing data in randomized clinical trials. Clin Trials 2005, 2(5):379-386.

28. Kenward MG, Molenberghs G: Last observation carried forward: a crystal ball? J Biopharm Stat 2009, 19(5):872-888.

29. Molnar FJ, Man-Son-Hing M, Hutton B, Fergusson DA: Have lastobservation-carried-forward analyses caused us to favour more toxic dementia therapies over less toxic alternatives? a systematic review. Open Medicine 2009, 3(2):1-20.

\section{doi:10.1186/1471-2288-14-118}

Cite this article as: Bell et al: Handling missing data in RCTs; a review of the top medical journals. BMC Medical Research Methodology 2014 14:118.

\section{Submit your next manuscript to BioMed Central and take full advantage of:}

- Convenient online submission

- Thorough peer review

- No space constraints or color figure charges

- Immediate publication on acceptance

- Inclusion in PubMed, CAS, Scopus and Google Scholar

- Research which is freely available for redistribution 